\title{
MOLECULAR DYNAMICS SIMULATION OF A WATER/METAL INTERFACE
}

\author{
E. SPOHR and K. HEINZINGER \\ Max-Planck-Institut für Chemie (Otto-Hahn-Institut), D-6500 Mainz, Federal Republic of Germany
}

Received 1 October 1985

\begin{abstract}
First results of a molecular dynamics study of a water/metal interface, lasting $3.3 \mathrm{ps}$ at an average temperature of $294 \mathrm{~K}$, are reported. The basic periodic box contains 216 water molecules and a crystal slab of 550 platinum atoms with (100) surface planes. A combination of a Lennard-Jones potential between centers of mass and a Coulomb potential arising from dielectric interactions of the water charge distribution with the metal is employed for the water-wall interaction, the ST2 model for the water-water, and a nearest-neighbour harmonic potential for the platinum-platinum interactions. Considerable adsorption at the interface together with a drastic change of the water structure is observed.
\end{abstract}

\section{Introduction}

Recent computer simulation studies of liquid water have focused on the behaviour of anisotropic systems. Both molecular dynamics (MD) and Monte Carlo (MC) simulations of water laminae [1-7] as well as MD calculations of a spherical water cluster [8] and of a lamina containing an aqueous Lil solution [9] have been performed. All authors used water-wall and ion-wall interactions to keep the anisotropic shape of the systems. Hence, with the exception of the work of Barabino et al. [4], who used image charge interactions (see below), the chosen potentials were one-dimensional with shallow minima resulting in an absence of specific adsorption of water at the interface. Accordingly the reported density profiles revealed only small density oscillations, these being mainly due to geometric packing effects. Anastasiou et al. [10] reported a short simulation of water in contact with a $\mathrm{NaCl}$ crystal and found a strong density increase near the crystal surface while there was only a weak indication of density oscillations extending further into the liquid phase.

In this work we present the first results of an MD simulation of a water lamina using the ST2 model [11] for water-water interactions and a wall model which is thought to describe a platinum single crystal in a first approximation. The wall model treats the metal as a polarizable single crystal and is described in detail in section 2 . In section 3 the density profiles of oxygen and hydrogen atoms are presented and section 4 discusses the effect of the metal walls on the oxygen-oxygen pair correlation functions.

\section{The model system and details of the simulation}

The rectangular basic box with sidelengths $L_{x}=$ $L_{y}=19.6 \AA$ and $L_{z}=42.1 \AA$ is shown in fig. 1 . The interval $-11.25<z<11.25 \AA$ is occupied by 216 ST2 water molecules and in the regions $11.25 \leqslant|z| \leqslant$ $21.05 \AA$ platinum atoms are located. As a consequence of the introduction of periodic boundary conditions the platinum can be regarded as one infinitely extended slab with a thickness of $19.6 \AA$ interacting on both surfaces with water molecules.

The values of $L_{x}$ and $L_{y}$ have been chosen in accordance with the periodicity of the face-centered cubic platinum lattice with lattice constant $a=3.92$ $\AA$. The crystal slab consists of $5 \times 5 \times 5$ face-centered cubic unit cells, with the (100) plane coinciding with the $X Y$ plane of the periodic box.

The water-wall interaction consists of two parts

$V_{\mathrm{H}_{2} \mathrm{O}-\text { wall }}=V_{\mathrm{LJ}}+V_{\mathrm{C}}$, 


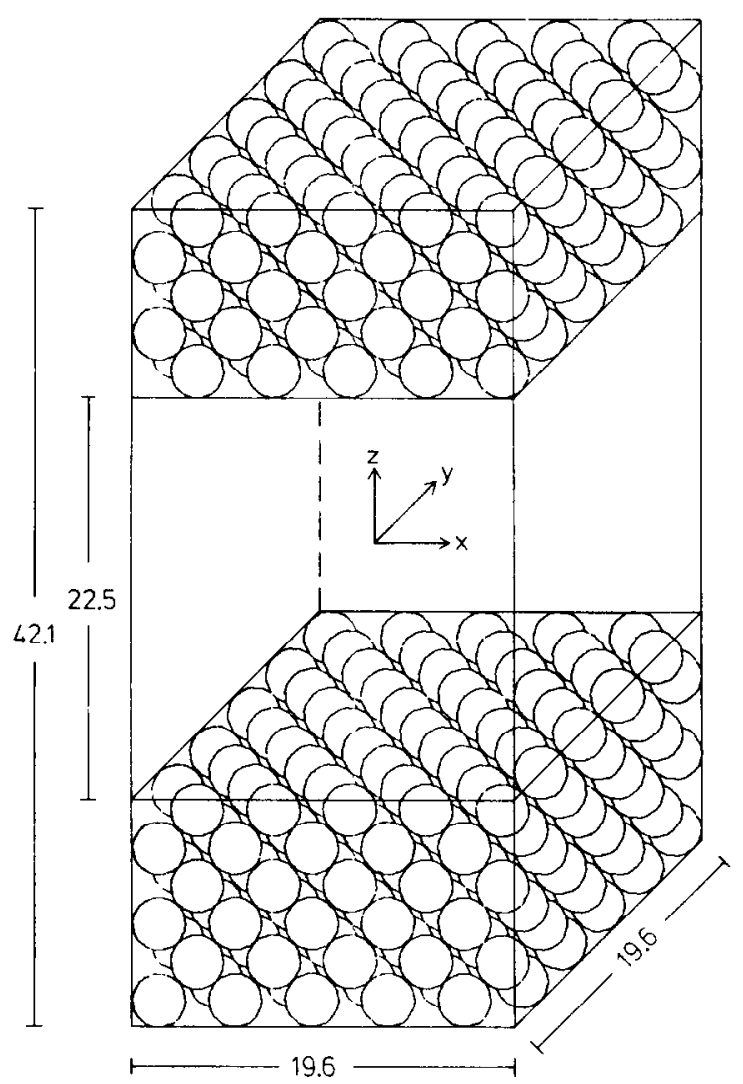

Fig. 1. Sketch of the basic tetragonal simulation cell. The water molecules are located in the centre of the box and the platinum atoms are represented by the circles. All distances are in $A$.

where $V_{\mathrm{LJ}}$ is a Lennard-Jones contribution arising from a pairwise summation of interactions between the oxygen atom of a water molecule and the platinum atoms in the crystal and $V_{\mathrm{C}}$ is a Coulomb part which is calculated from treating the interface as a dielectric discontinuity.

The $\mathbf{L}$ part can be written as

$$
V_{\mathrm{LJ}}=\sum_{k} 4 \epsilon\left[\left(\sigma / r_{k}\right)^{12}-\left(\sigma / r_{k}\right)^{6}\right] \text {, }
$$

with $\epsilon=9.57 \times 10^{-21} \mathrm{~J}$ and $\sigma=2.89 \AA . r_{k}$ is the distance between the oxygen atom of the water molecule and the platinum atom $k$ and the summation goes over all Pt atoms inside a cutoff radius of $L_{x} / 2$. The parameters $\epsilon$ and $\sigma$ have been calculated by applying Kong's combination rule [12] to the oxygenoxygen $\mathrm{L}$ potential of the TIPS water model by
Jorgensen [13] and a Pt-Pt LJ potential by Halicioglu and Pound [14] which has been used before in computer simulations of surface diffusion of $\mathrm{C}$ and $O$ atoms on Pt surfaces [15]. The oxygenoxygen potential has not been taken from the ST2 model because due to the switching function in this model the LJ parameter $\epsilon$ seems to be too small.

The Coulomb part $V_{C}$ is calculated by treating the interface as a dielectric discontinuity between a vacuum with embedded point charges (solution) and a homogeneous dielectric medium of relative permittivity $\epsilon_{\mathrm{r}}$. This electrostatic boundary-value problem can be solved using the method of image charges (see, e.g., ref. [16]). If the two interfaces are not coupled due to the cut-off truncation of interactions (see also discussion in ref. [4]) every charge $q_{l}$ in the solution induces an image charge of magnitude

$Q_{l}=\frac{1-\epsilon_{\mathrm{r}}}{1+\epsilon_{\mathrm{r}}} q_{l}$

in the metal at a position determined by reflecting the real charge at the discontinuity. The reflexion plane has been chosen as the plane of first-layer lattice atoms. For a grounded metal, in order to have zero electrostatic potential at the interface, $\epsilon_{\mathrm{r}}$ must be infinite yielding image charges $Q_{l}=-q_{l}$. Every charge in the solution interacts with all image charges by means of a Coulomb potential. Hence, the Coulomb part is given by

$V_{\mathrm{C}}=-\sum_{\alpha=1}^{4} \sum_{\beta} q_{\alpha} q_{\beta} / r_{\alpha \beta}$,

where the first sum is over the charges $q_{\alpha}$ of the water molecule of interest and the second sum is over all image charges inside the cut-off sphere. $r_{\alpha \beta}$ is the distance between the charge $q_{\alpha}$ and the image charge of $q_{\beta}$.

The water-wall potential in this form describes several features of a metal/water interface. The Coulomb potential leads to a reduction of surface roughness of the metal which is in accordance with scattering experiments on metal surfaces where a smaller corrugation is found than on other surfaces due to the collective effects of the electron gas (see, e.g., ref. [17]). Secondly the metal is instantaneously polarizable due to the image charge part of the interaction.

Furthermore, the metal crystal itself has vibra- 
tional degrees of freedom. Only nearest neighbours interact through a harmonic potential with a force constant $k=4.66 \times 10^{-9} \mathrm{~J} / \AA[18]$. This allows for coupling of lattice motions to the hindered translational and librational motion of wa ter molecules. A one-constant model is assumed to give an adequate description of the vibrational motions of bulk platinum [18].

Using this model the simulation extended at an average temperature of $294 \mathrm{~K}$ over 3.3 ps after a 3 ps equilibrium phase starting from the final configuration of a 20 ps run of pure water between LJ walls [5]. All interactions were truncated with a cut-off radius of $9.8 \AA$ and the shifted force method [19] has been employed.

\section{Density profiles}

The oxygen and hydrogen density profiles are shown in fig. 2 together with those of a simulation of ST2 water between Lennard-Jones walls [5]. The oxygen profile shows much stronger oscillations than reported in previous work $[1-8]$. The density in the first layer is approximately six times as high as the

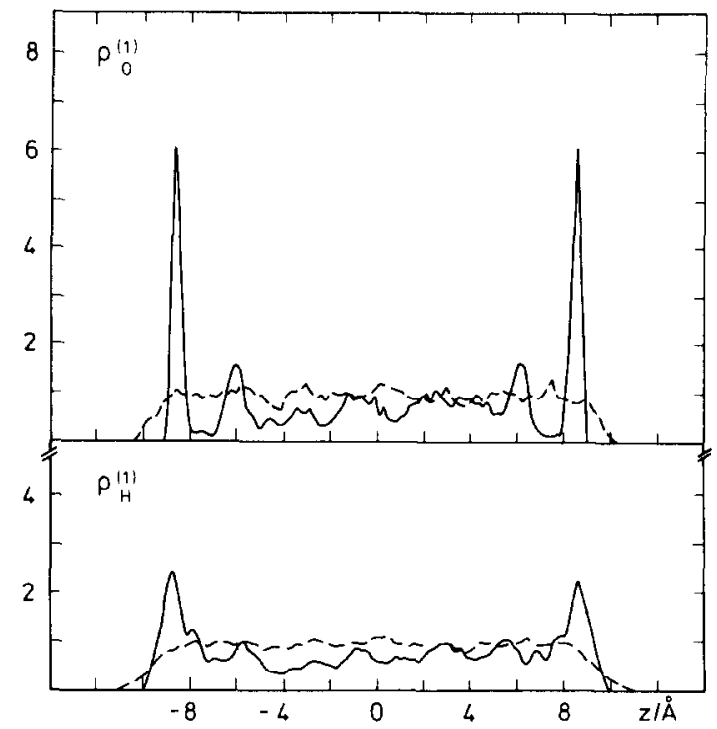

Fig. 2. Relative oxygen (top) and hydrogen (bottom) density profiles from the present simulation (full lines) and for a simulation of ST2 water between Lennard-Jones walls [5]. The relative density is one if the number density corresponds to that of bulk water. bulk water density, indicating strong adsorption of water at the interface. This result is confirmed by the calculated adsorption energy of $34 \mathrm{~kJ} / \mathrm{mole}$. It is calculated at the minimum of the simulation-averaged distance-dependent water-wall interaction energy and occurs at a distance of $2.31 \AA$ from the wall. The second oxygen peak is still quite pronounced and partially due to direct water-wall attraction and partially due to water-water interaction with water molecules of the strongly ordered surface layer as can be seen from the shape of the oxygen-oxygen pair correlation functions (see below).

The hydrogen density profile shows a broader and less pronounced first-layer peak and there is some indication for density oscillations extending over the whole lamina. The hydrogen density also shows stronger oscillations than in previously reported simulations, except that of Barabino et al. [4] who also used an image-charge potential for a relative permittivity $\epsilon_{\mathrm{r}}=3$. The significantly different results for the ratio of the oxygen to the hydrogen peak height in this work and in ref. [4] might be a consequence of the different water models employed, because the charge distribution, and consequently the relative magnitude of the forces on oxygen and hydrogen atoms, is different in the ST2 model and in the MCY model [20] used in ref. [4].

\section{Oxygen-oxygen pair correlation functions}

In addition to the distinct effect of the water adsorption on the density profiles, a strong influence on the center-of-mass liquid structure can be detected. Fig. 3 shows the oxygen-oxygen pair correlation functions (PCFs) for the following three subsystems: One oxygen is located in the surface layer $(|z|>7.4 \AA$; full line), in the second layer $(5.5<|z| \leqslant 7.4 \AA$; dotted line), and in the interior part of the lamina $(|z| \leqslant 5.5 \AA$; dashed line). The structure around water molecules in the surface layer and around the molecules in the interior of the lamina show pronounced differences. The structure around the second-layer molecules seems to have properties intermediate between the two aforementioned ones, owing to the fact that in this region the transition of normal bulk water structure (with more or less tetrahedral symmetry) to the crystal-induced surface 


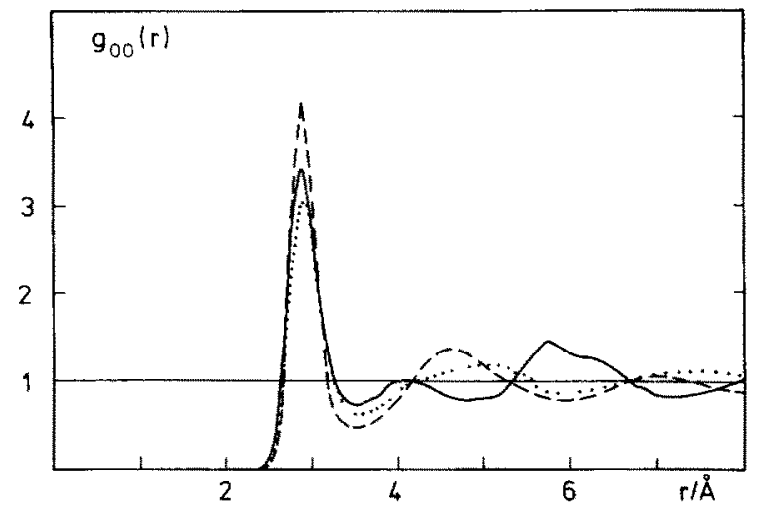

Fig. 3. Oxygen -oxygen pair correlation functions for an oxygen located in the region $|z|>7.4 \AA$ (full line), $5.5<$ $|z|<7.4 A$ (dotted line), and $|z|<5.5 \mathrm{~A}$ (dashed line).

structure (with quadratic symmetry) has to take place.

The PCF in the interior of the lamina has a shape similar to that of pure bulk water $[11,21]$ except for the fact that the height of the first maximum is increased. This seems to be a consequence of a more tetrahedral structure in the interior of the lamina induced by the lower density in this region as compared to bulk water. This tendency seems to be generally quite pronounced in ST2 water as an increase in peak height due to a lower local density has also been found in previous MD simulations $[5,9]$. The increased peak height is also in accordance with the more favorable nearest-neighbour pair interaction energy and with the distribution of pair interaction energies which shows more favorable and less unfavorable pairs than in normal bulk water.

The wall layer O-O PCF (full line) resembles clearly the surface structure of the platinum crystal slab. Due to the fact that with $2.77 \AA$ the Pt-Pt dis-

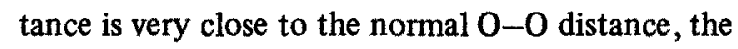
first peak height in the PCF remains approximately constant. Contrarily, the second water peak - usually at about $4.7 \AA$ - vanishes completely. Instead, two peaks at 4.2 and $\approx 6 \AA$ occur. As two adjacent potential energy minima for a water molecule on the sur- face are $2.77 \AA$ apart due to their quadratic arrangement, minima also occur at $2^{1 / 2}$ and at 2 times this distance. Hence, the two observed maxima in the PCFs are a consequence of the competition between adsorption on the surface and water-water interactions leading to somewhat greater distances for the actual occurrence of the maxima.

\section{References}

[1] B. Jönsson, Chem. Phys. Letters 82 (1981) 520.

[2] N.I. Christou, J.S. Whitehouse, D. Nicholson and N.G. Parsonage, Faraday Symp. Chem. Soc. 16 (1981) 139.

[3] M. Marchesi, Chem. Phys. Letters 97 (1983) 224.

[4] G. Barabino, C. Gavotti and M. Marchesi, Chem. Phys. Letters 104 (1984) 478 .

[5] R. Sonnenschein and K. Heinzinger, Chem. Phys. Letters 102 (1983) 550.

[6] C.Y. Lee, J.A. McCammon and P.J. Rossky, J. Chem. Phys. 80 (1984) 4448.

[7] N.I. Christou, J.S. Whitehouse, D. Nicholson and N.G. Parsonage, Mol. Phys. 55 (1985) 397.

[8] R.M. Townsend, J. Gryko and S.A. Rice, J. Chem. Phys. 82 (1985) 4391.

[9] E. Spohr and K. Heinzinger, J. Chem. Phys., to be published.

[10] N. Anastasiou, D. Fincham and K. Singer, J. Chem. Soc. Faraday Trans. II 79 (1983) 1639.

[11] F.H. Stillinger and A. Rahman, J. Chem. Phys. 60 (1974) 1545.

[12] C.L. Kong, J. Chem. Phys. 59 (1973) 2464.

[13] W.L. Jorgensen, J. Chem. Phys. 77 (1982) 4156.

[14] T. Halicioglu and G.M. Pound, Phys. Stat. Sol. 30a (1975) 619.

[15] J.D. Doll and D.L. Freeman, Surface Sci. 134 (1983) 769.

[16] J.D. Jackson, Classical electrodynamics (Wiley, New York, 1962).

[17] T. Engel and K.-H. Rieder, in: Structural studies of surfaces, ed. G. Höhler, Springer Tracts in Modern Physics 91 (Springer, Berlin, 1982) pp. 157 ff.

[18] J.E. Black and P. Bopp, Surface Sci. 140 (1984) 275.

[19] W.B. Streett, D.J. Tildesley and G. Saville, ACS Symp. Ser. 86 (1978) 144.

[20] O. Matsuoka, E. Clementi and M. Yoshimine, J. Chem. Phys. 64 (1976) 1351.

[21] Gy.I. Szász, K. Heinzinger and W.O. Riede, Z. Naturforsch. 36a (1981) 1067. 